\title{
MRI/TRUS FUSION guided biopsy as first approach in ambulatory setting: Feasibility and performance of a new fusion device
}

\author{
Daniele D'Agostino ${ }^{1}$, Federico Mineo Bianchi ${ }^{2}$, Daniele Romagnoli ${ }^{1}$, Marco Giampaoli ${ }^{1}$, Paolo Corsi ${ }^{1}$, \\ Alessandro Del Rosso ${ }^{1}$, Riccardo Schiavina ${ }^{2}$, Eugenio Brunocilla ${ }^{2}$, Angelo Porreca ${ }^{1}$ \\ ${ }^{1}$ Department of Robotic Urological Surgery, Abano Terme Hospital, Abano Terme, Italy; \\ 2 Department of Urology, University of Bologna, Bologna, Italy.
}

\begin{abstract}
Summary Purpose: To evaluate the detection rate of Magnetic Resonance Imaging/Transrectal Ultrasound (MRI/TRUS) Fusion Biopsy performed in a series of patients with suspicious prostate cancer in an ambulatory setting.

Materials and methods: Between March 2018 and January 2019 a series of 155 patients undergoing MRI/TRUS fusionguided biopsy were prospectively enrolled. All patients presented a suspected diagnosis for prostate cancer because of raised Prostate Specific Antigen (PSA) serum level and/or abnormal physical examination (digital rectal examination), and showed at least one suspicious area at the multiparametric Magnetic Resonance Imaging (mpMRI).

Results: Of 155 patients, 58 (37.4\%) were biopsy-naïve, 97 (62.6\%) had at least 1 previous negative TRUS-guided biopsy. The median age of the patient cohort was 66 years (IQR, 6169); the median prebiopsy PSA value was $7.1 \mathrm{ng} / \mathrm{ml}$ (IQR, 58.9). Overall, the Fusion-TB findings were positive in 94 of 155 patients with a detection rate (DR) of 60\%; a significantly high DR was obtained in terms of clinically significant prostate cancer (csPCa) by Fusion-TB (61 pts; 41.9\%). The overall DR in the 121 biopsy-naive patients was $60.6 \%$. In the subgroup of the 34 patients with at least 1 previous set of TRUS-GB, overall DR was $39.3 \%$ (35/50).

Conclusions: The targeted MRI/TRUS fusion-guided biopsy represents a safe and accurate approach for diagnosis of csPCa, especially in patient with previous TRUS guided biopsy negative and suspicious prostate cancer.
\end{abstract}

KEY WORDS: Prostate cancer; Magnetic Resonance; Prostate biopsy.

Submitted 8 July 2019; Accepted 2 August 2019

\section{INTRODUCTION}

Prostate cancer (PCA) is the most frequent among solid tumors in the male sex, as emerges from the 2016 estimate of the American Cancer Society (1). Currently, diagnosis of PCA is one of the most debated topics in the urology literature (2) and is based on the serum dosage of prostate specific antigen (PSA) and digital rectal exploration. PSA levels can be elevated not only in the case of prostatic carcinoma but also in the case of other pathologies, such as benign prostatic hyperplasia and inflammatory states of different nature (3) with consequent risk of over-diagnosis and over-treatment. Although the intro- duction of PSA has radically changed the diagnosis of PCA, as amply highlighted by the literature, it is a test that has limits in terms of sensitivity and specificity; for example about $15 \%$ of men with PSA levels equal to or below $4.0 \mathrm{ng} / \mathrm{ml}$ are affected by prostatic carcinoma that in about $15 \%$ of cases is of high-grade (4). To date, the conventional diagnosis of prostatic carcinoma is performed by identifying histopathology on systematic ultrasound-guided biopsy specimens with a sensitivity of $45-70 \%$ for clinically significant PCA (5). The main disadvantages related to this method are the loss of identification of a substantial portion of patients with significant prostatic carcinoma (about 20\%) linked to sampling errors, in particular at the level of the anterior prostate area (6) and the possibility of important complications following the procedure, above all related to sampling performed by trans-rectal ultrasound-guided biopsy (7). The actually limited detection rate represents an important concern and the management of patients with persistent suspected diagnosis of PCA and previous set of negative biopsies represents a continuing challenge. Magnetic Resonance Imaging (MRI) has shown a high sensitivity and specificity in diagnosing clinically significant PCA (csPCa) $(5,6)$ and, exploiting the use of functional studies, multiparametric MRI (mpMRI) improves the identification of PCA lesion within the gland $(7,8)$. A growing body of evidence suggests that mpMRI, improving the risk classification of lesions, could reduce false-negative rates and the necessity of repeat biopsies in presence of suspected PCA $(8,9)$; not surprisingly, a MRI targeted biopsy should be strongly applied for any patient with a prior negative set of prostate biopsy who has persistent clinical suspected diagnosis for PCA as reported by recent AUA Consensus Statement (9); more frequently MRI is used in the first diagnostic phase for PCA; for this reason the MRI-guided approach of prostatic biopsy is increasingly used. Approaches for targeted biopsy include visual estimation TRUS-GB (cognitive technique), software co-registered MRI-ultrasound fusion (fusion technique) and in-bore MRI-guided biopsy (MRI-GB). Studies from literature suggest that there was no significant advantage of MRI-GB compared with fusion technique concerning overall 
detection rate and clinical significant neoplasm detection rate (10) even if the main potential limitation of MRI-GB consist of impossibility to perform a simultaneous standard TRUS biopsy, especially in biopsy naive patients, since up to $16 \%$ of men with no suspicious mpMRI could reveal csCaP on systematic biopsy (11). Different software for MRI/TRUS fusion-guided biopsy systems are at the present time used: the aim of this study was to evaluate the detection rate of MRI TRUS Fusion Biopsy performed in a series of patients with suspicious PCA in ambulatory setting with a new fusion device.

\section{MATERIALS AND METHOdS}

\section{Patients cohort and methodology}

Between March 2018 and January 2019 a series of 155 patients undergoing MRI/TRUS fusion-guided biopsy were prospectively enrolled. All patients had a suspected diagnosis for PCA because of elevated value of Prostate Specific Antigen (PSA) serum level and/or abnormal physical examination (digital rectal examination), and showed at least one suspicious area at the mpMRI. According to the European Society of Urogenital Radiology (ESUR) guidelines, the presence of csPCa at mpMRI was defined equivocal, likely or highly likely basing on the PIRADS-v2 (Prostate Imaging Reporting and Data Systemversion 2) score of $3 / 5,4 / 5$ or $5 / 5$, respectively; the localization of index lesion are reported in Table 2 (12).

This study was conducted under the approval of local Institutional Review Board and in the accordance with good clinical practice guidelines and the ethical principles of the Declaration of Helsinki.

Primary endpoints of this study were overall detection rates of PCA (PCA DR), csPCa Detection Rate (csPCa DR). Secondary endpoints were correlations between mpMRI parameters biopsy results, comparison to definitive pathologic results of surgical specimens (when available) and complications rates.

\section{Multiparametric Magnetic Resonance Imaging and analysis}

MRI examination and analysis. All the MRI examinations were performed with a 32 channels $1.5 \mathrm{~T}$ whole body scanner (Achieva XR; Philips Medical Systems, Best, Netherlands) with a 32-channels phased-array surface coil without endorectal coil. After local three-plane acquisition, required for the correct positioning of the sequences, the morphological and functional studies were carried out. Morphological study of the prostate gland were obtained with Turbo Spin Echo (TSE) T2weighted sequences (TE $100 \mathrm{msec}$, TR $4074 \mathrm{msec}$, Slice Thickness $3 \mathrm{~mm}$, Slice Spacing $0.3 \mathrm{~mm}$, Field of View FOV $180 \times 180 \mathrm{~mm}$ and matrix size $276 \times 205$ ) in the sagittal, axial and coronal planes, including seminal vesicles and the entire prostate gland. For the functional study, DWI, DCE-MRI and MRS acquisition were performed. The DWI acquisition was carried out in the axial plane, using a single-shot echo-planar imaging (SSEPI) sequence, with three b-values $\left(0,600\right.$ and $\left.1500 \mathrm{~s} / \mathrm{mm}^{2}\right)$, slice thickness of $3 \mathrm{~mm}$, FOV $180 \times 180 \mathrm{~mm}$ and matrix size $80 \times 71$. The DCE-MRI was obtained using three- dimensional (3D) T1W High Resolution Isotropic Volume Examination (THRIVE) sequence during the intravenous injection of a contrast bolus of $0.1 \mathrm{mmol}$ per kilogram of body weight of Meglumine gadobenate (Multihance, Bracco Diagnostics, Milan, Italy), at flow rate of $3.5 \mathrm{ml} / \mathrm{sec}$ followed by $15 \mathrm{ml}$ of saline solution.

\section{Conduct of the biopsy}

The biopsies were performed within three weeks from the first diagnostic mpMRI study by a single urologist with a consolidated experience in MRI Fusion-GB.

All patients received oral antibiotic prophylaxis with quinolones (Ciprofloxacin $500 \mathrm{mg}$ ) twice a day, started the day before the procedure and prolonged for at least 2 days thereafter. Prostate biopsy procedures were conducted in an ambulatory setting with patient in lithotomic position. Peri-prostatic nerve blockade local anesthesia with lidocaine $2 \%$ was administered immediately before biopsy to each patient. Biopsies were conducted using a disposable biopsy gun with a 18-gauge needle and an Ultrasound Platform (BK ultrasound 5000) with a biplanar probe. Using the BK Ultrasound 5000 MRI-TRUS Fusion platform, Fusion-TB was performed on the previously identified suspicious area at the mpMRI using a real time alignment of the T2-weighted sequence to the TRUS image. MRI-TRUS images alignment was possible due to a tracking device consisted in a sensor coil on the TRUS probe paired with a magnetic field generator in order to register the location of the tracking device in the 3D space. At least 3 cores were taken for each lesion and the number of additional cores were based on the diameter of the lesion. The number of cores taken was related to the size of the lesions; the cores were carried out along the long axis of the lesion with a maximum of two biopsies taken for each needle. TRUS Standard Biopsy was a typical 12 cores double sextant template from lateral to medial of base, mid and apex. Only the TRUS images, with no mp-MRI target data available, were used for the standard biopsy portion of the case. After the procedure, patients were observed for 1 hour and were re-evaluated by outpatient visit after 7-10 days in order to record any potential complication.

\section{Statistical analysis}

Continuous variables were reported as medians with interquartile ranges (IQR) whereas categorical variables were described as frequencies with percentages.

The Mann-Whitney and Pearson Chi-square test were used to compare medians and frequencies among patients with positive and negative biopsies, respectively. Uni- and multivariate logistic regression models with enter method were used to identify which covariates could predict PCA, csPCa and concordance between mpMRI index lesion and bioptic index lesion. An alpha value of 5\% was set to be the threshold to determine statistical significance.

Statistical analyses were conducted using SPSS ${ }^{\circledR}$ v21 (IBM Corp, Armonk, NY) for Macintosh ${ }^{\circledR}$.

\section{Results}

The clinical, radiologic, and pathologic characteristics of the entire population are listed in Table 1. Of 155 patients, 58 (37.4\%) were biopsy-naive, 97 (62.6\%) had 
at least 1 previous negative set of random TRUS-GB. The median age of the patient population was 66 years (IQR, 61-69) and median prebiopsy PSA level was $7.1 \mathrm{ng} / \mathrm{ml}$ (IQR, 5-8.9). The median number of targeted biopsies per patient was 4 (IQR, 3-4), and, accordingly, the median total number of biopsies including the standard 12-cores was 14 (IQR,12-15). At the prebiopsy mpMRI study, a total of 155 suspected lesions were identified and were scheduled for Fusion-TB. The median diameter of the index lesion was $13 \mathrm{~mm}$ (IQR, 9-18.1 mm). The univariate logistic regression model showed a crucial role of V2PIRADS score of index lesion in the prediction of PCA and csPCA (Tables 3, 4). Overall, the FUSION-TB findings were positive in 94 of 155 patients with a DR of $60 \%$; a significantly high DR was obtained in terms of clinically significant PCA (csPCa) of Fusion-TB (61 pts; 41.9\%). The overall DR in the 121 biopsy-naive patients was $60.6 \%$. In the subgroup of the 34 patients with at least 1

Table 1.

Design of studies of Serenoa repens for BPH treatment.

\begin{tabular}{|c|c|c|c|c|}
\hline & $\begin{array}{l}\text { Overall population } \\
\quad(n=155)\end{array}$ & $\begin{array}{c}\text { Biopsy+patients } \\
\quad(\mathrm{n}=94)\end{array}$ & $\begin{array}{l}\text { Biopsy-patients } \\
\quad(\mathrm{n}=61)\end{array}$ & p-value \\
\hline \multicolumn{5}{|l|}{ Age } \\
\hline Median & 66 & 66 & 65 & 0.5 \\
\hline IQR & $61-69$ & $61-70$ & $61-69$ & \\
\hline \multicolumn{5}{|c|}{$\mathrm{PSA}(\mathrm{ng} / \mathrm{ml})$} \\
\hline Median & 7.1 & 7.2 & 7 & 0.9 \\
\hline IQR & $5-8.9$ & $5.4-9.3$ & $4.6-8.8$ & \\
\hline \multicolumn{5}{|c|}{ PSA density $\left(\mathrm{ng} / \mathrm{ml}^{\prime} / \mathrm{cm}^{3}\right)$} \\
\hline Median & 0.12 & 0.13 & 0.11 & 0.03 \\
\hline IQR & $0.09-0.16$ & 0.1-0.17 & $0.08-0.13$ & \\
\hline \multicolumn{5}{|c|}{ Prostate Volume $\left(\mathrm{cm}^{3}\right)$} \\
\hline Median & 55 & 50 & & 0.2 \\
\hline IQR & $43-75$ & $40-60$ & $46-80$ & \\
\hline \multicolumn{5}{|c|}{ Previous TRUS-GB (\%) } \\
\hline No & $121(78.1)$ & $57(60.6)$ & $40(65.6)$ & 0.6 \\
\hline Yes & $34(21.9)$ & $37(39.3)$ & $21(34.4)$ & \\
\hline \multicolumn{5}{|c|}{ Index lesion diameter (mm) } \\
\hline Median & 13 & 11 & 13 & 0.9 \\
\hline IQR & $9-18.1$ & $8-16$ & $9-16.5$ & \\
\hline \multicolumn{5}{|c|}{ Index lesion location (\%) } \\
\hline Anterior & $44(28.4)$ & $27(28.7)$ & $17(27.9)$ & 0.7 \\
\hline Posterior & $111(71.6)$ & $67(71.3)$ & $44(72.1)$ & \\
\hline \multicolumn{5}{|c|}{ Index lesion site (\%) } \\
\hline Peripheral & $107(69)$ & $65(69.1)$ & $42(68.9)$ & 0.1 \\
\hline Central & $48(31)$ & $29(28.7)$ & $19(31.1)$ & \\
\hline \multicolumn{5}{|c|}{ Index lesion PIRADS V2 score (\%) } \\
\hline 3 & $72(46.5)$ & $29(38.5)$ & $43(70.5)$ & $<0.001$ \\
\hline 4 & $53(34.2)$ & $41(38.5)$ & $12(19.7)$ & \\
\hline 5 & $30(19.4)$ & $24(23.1)$ & $6(9.8)$ & \\
\hline \multicolumn{5}{|c|}{ Total cores taken per patient } \\
\hline Median & 14 & 14 & 13 & 0.96 \\
\hline IQR & $12-15$ & $12-15$ & $12-15$ & \\
\hline \multicolumn{5}{|c|}{ Target biopsy cores taken per patient } \\
\hline Median & 4 & 4 & 4 & 0.9 \\
\hline IQR & 3-4 & $3-5$ & $3-4$ & \\
\hline \multicolumn{5}{|c|}{ ISUP grade group (\%) } \\
\hline Negative & $61(39.4)$ & $0(0)$ & $61(100)$ & - \\
\hline 1 & $29(18.7)$ & $29(18.7)$ & $0(0)$ & \\
\hline 2 & $26(16.8)$ & $26(16.8)$ & $0(0)$ & \\
\hline 3 & $28(18.1)$ & $28(18.1)$ & $0(0)$ & \\
\hline 4 & $11(7.1)$ & $11(7.1)$ & $0(0)$ & \\
\hline 5 & $0(0)$ & $0(0)$ & $0(0)$ & \\
\hline
\end{tabular}

Table 2.

Localization of Index lesion at mpMRI and bioptic results stratified according to previous biopsy status and bioptic findings.

\begin{tabular}{|c|c|c|c|}
\hline & $\begin{array}{c}\text { Biopsy+patients } \\
\quad(\mathrm{n}=94)\end{array}$ & $\begin{array}{c}\text { Biopsy-patients } \\
\quad(n=61)\end{array}$ & p-value \\
\hline \multicolumn{4}{|l|}{ Biopsy näive patients ( $n=97)$} \\
\hline \multicolumn{4}{|c|}{ Index Lesion Location (\%) } \\
\hline Anterior & $15(26.3)$ & $13(32.5)$ & 0.7 \\
\hline Posterior & $42(73.7)$ & $27(67.5)$ & \\
\hline \multicolumn{4}{|c|}{ Index lesion site (\%) } \\
\hline Central & $17(29.8)$ & $15(37.5)$ & 0.3 \\
\hline Peripheral & $40(70.2)$ & $32(62.5)$ & \\
\hline \multicolumn{4}{|l|}{ Previous negative biopsies $(n=58)$} \\
\hline \multicolumn{4}{|c|}{ Index Lesion Location (\%) } \\
\hline Anterior & $12(32.4)$ & $4(19)$ & 0.4 \\
\hline Posterior & $25(67.6)$ & 17 (81) & \\
\hline \multicolumn{4}{|l|}{ Index lesion site (\%) } \\
\hline Central & $12(32.4)$ & $4(19)$ & 0.4 \\
\hline Peripheral & $25(67.6)$ & $17(81)$ & \\
\hline
\end{tabular}

Table 3.

Uni-variate logistic regression model predicting PCa $(n=94)$ at Fusion biopsy.

\begin{tabular}{|c|c|c|}
\hline \multicolumn{3}{|c|}{ Univariate analysis } \\
\hline & HR (95\% Cl) & p-value \\
\hline Age (yrs) & $1.01(0.97-1.07)$ & 0.5 \\
\hline PSA (ng/ml) & $1.08(0.97-1.19)$ & 0.2 \\
\hline $\begin{array}{l}\text { PSA density }\left(\mathrm{ng} / \mathrm{ml} / \mathrm{cm}^{3}\right) \\
<0.15 \\
0.15\end{array}$ & $\begin{array}{c}\text { Ref. } \\
0.94(0.47-1.87)\end{array}$ & 0.9 \\
\hline $\begin{array}{l}\text { Previous TRUS-GB } \\
\text { No } \\
\text { Yes }\end{array}$ & $\begin{array}{c}\text { Ref. } \\
1.24(0.63-2.42)\end{array}$ & 0.5 \\
\hline $\begin{array}{l}\text { Index Lesion Site } \\
\text { Peripheral } \\
\text { Central }\end{array}$ & $\begin{array}{c}\text { Ref. } \\
0.99(0.49-1.98)\end{array}$ & 0.97 \\
\hline $\begin{array}{l}\text { Lesion Location } \\
\text { Posterior } \\
\text { Anterior }\end{array}$ & $\begin{array}{c}\text { Ref. } \\
0.98(0.47-1.96)\end{array}$ & 0.9 \\
\hline $\begin{array}{l}\text { Index lesion PiRADS V2 score } \\
3 \\
4 \\
5\end{array}$ & $\begin{array}{c}\text { Ref. } \\
5.07(2.28-11.24) \\
5.93(2.16-16.3)\end{array}$ & $\begin{array}{c}<0.001 \\
0.001\end{array}$ \\
\hline Index lesion diameter (mm) & $1.01(0.96-1.07)$ & 0.7 \\
\hline $\mathrm{N}$ of cores taken & $1(0.86-1.16)$ & 0.99 \\
\hline $\mathrm{N}$ of target cores taken & $1.07(0.72-1.58)$ & 0.8 \\
\hline
\end{tabular}

previous set of TRUS-GB, overall DR was 39.3\% (35/50). In the series of biopsy naive patients whose clinically significant PCA (csPCa) was found, the location of index lesion was anterior in 26.3\% (15/57 cases) while we observed a posterior lesion in $73.7 \%$ (42/57 cases).

In patients with previous negative TRUS-GB whose csPCa was diagnosed the location of index lesion was anterior in $32.4 \%$ (12/37 cases) and posterior in 67.5\% (25/37 cases). Overall, in the patients with a PI-RADS-v2 score of 3 of 5 , 4 of 5 , and 5 of 5 , DR for PCAa were $40.2 \%$ (29/72), $77.0 \%(41 / 53)$ and $80.0 \%(24 / 30)$, respectively ( $p<$ 0.001 ). In Table 5 are reported univariate logistic regres- 
Table 4.

Univariate logistic regression model predicting csPCa $(n=54)$ at fusion biopsy.

\begin{tabular}{|c|c|c|}
\hline & Univariate analysis & \\
\hline & HR (95\% Cl) & $p$-value \\
\hline Age (yrs) & $1.02(0.97-1.07)$ & 0.2 \\
\hline PSA (ng/ml) & $1.03(0.96-1.1)$ & 0.4 \\
\hline $\begin{array}{l}\text { PSA density }\left(\mathrm{ng} / \mathrm{ml} / \mathrm{cm}^{3}\right) \\
<0.15 \\
\geq 0.15\end{array}$ & $\begin{array}{c}\text { Ref. } \\
1.08(0.55-2.12)\end{array}$ & 0.8 \\
\hline $\begin{array}{l}\text { Previous TRUS-GB } \\
\text { No } \\
\text { Yes }\end{array}$ & $\begin{array}{c}\text { Ref. } \\
1.57(0.82-3.02)\end{array}$ & 0.2 \\
\hline $\begin{array}{l}\text { Index Lesion Site } \\
\text { Peripheral } \\
\text { Central }\end{array}$ & $\begin{array}{c}\text { Ref. } \\
0.67(0.33-1.33)\end{array}$ & 0.3 \\
\hline $\begin{array}{l}\text { Index lesion PiRADS V2 score } \\
3 \\
4 \\
5\end{array}$ & $\begin{array}{c}\text { Ref. } \\
3.42(1.63-7.22) \\
4.86(1.95-12.11)\end{array}$ & $\begin{array}{l}0.001 \\
0.001\end{array}$ \\
\hline Index lesion diameter (mm) & $1.01(0.96-1.07)$ & 0.6 \\
\hline N of cores taken & $1.03(0.89-1.19)$ & 0.7 \\
\hline $\bar{N}$ of target cores taken & $1.09(0.74-1.6)$ & 0.7 \\
\hline
\end{tabular}

\section{Table 5.}

Univariate logistic regression model predicting concordance between Index lesion at mpMRI and highest cGS in the bioptic cores.

\begin{tabular}{|c|c|c|c|c|}
\hline & \multicolumn{2}{|c|}{ Univariate analysis } & \multicolumn{2}{|c|}{ Multivariate analysis } \\
\hline & HR $(95 \%$ CI) & p-value & HR $(95 \%$ CI) & p-value \\
\hline Age (yrs) & $1.03(0.96-1.1)$ & 0.5 & - & - \\
\hline PSA (ng/ml) & $1.05(0.92-1.2)$ & 0.4 & - & - \\
\hline \multicolumn{5}{|l|}{ PSA density $\left(\mathrm{ng} / \mathrm{ml} / \mathrm{cm}^{3}\right)$} \\
\hline$<0.15$ & Ref. & 0.5 & - & - \\
\hline 0.15 & $1.45(0.51-4.15)$ & & & \\
\hline \multicolumn{5}{|l|}{ Previous TRUS-GB } \\
\hline No & Ref & 0.1 & - & - \\
\hline Yes & $2.2(0.77-6.23)$ & & & \\
\hline \multicolumn{5}{|l|}{ Index lesion site } \\
\hline Peripheral & Ref. & 0.01 & Ref. & 0.02 \\
\hline Central & $0.29(0.11-0.77)$ & & $0.3(0.1-0.82)$ & \\
\hline \multicolumn{5}{|l|}{ Index lesion location } \\
\hline Posterior & Ref. & 0.8 & - & - \\
\hline Anterior & $0.84(0.29-2.43)$ & & & \\
\hline \multicolumn{5}{|c|}{ Index lesion PiRADS V2 score } \\
\hline 3 & Ref. & & Ref. & \\
\hline 4 & $0.74(0.26-2.08)$ & 0.6 & $0.75(0.25-2.2)$ & 0.6 \\
\hline 5 & $8.76(1.01-76.08)$ & 0.05 & $8.83(0.99-78.93)$ & 0.05 \\
\hline \multicolumn{5}{|l|}{ Index lesion diameter } \\
\hline$(\mathrm{mm})$ & $1.02(0.95-1.1)$ & 0.6 & - & - \\
\hline $\mathrm{N}$ of cores taken & $1.07(0.87-1.31)$ & 0.5 & - & - \\
\hline $\mathrm{N}$ of target cores taken & $0.89(0.49-1.64)$ & 0.7 & - & - \\
\hline
\end{tabular}

sion model predicting concordance between Index lesion at mpMRI and highest Gleason score in the bioptic cores.

\section{Discussion}

Several major changes have taken place in the last decade regarding the diagnosis of PCA; the introduction of new imaging techniques (traditional radiology, nuclear medicine, etc.) is radically changing the approach to the patient with PCA (13-17). In particular the most important innovation was represented by the introduction of multiparametric magnetic resonance imaging (mpMRI) in the diagnosis and management of PCA (active surveillance, surgery, radiotherapy, etc.) $(18,19)$.

In fact, this examination showed an elevate detection and localization rate of csPCa thus making it possible to perform selected targeted biopsies instead of systematic ultrasound guided biopsies. In any case, TRUS-GB represents the "gold-standard" technique of histological diagnosis of PCA although MRI-targeted biopsies (cognitive, fusion and "in bore" technique) significantly increased the detection of high risk PCA while decreasing the detection of low risk PCA compared with standard biopsy; moreover a lower number of cores could be required in men with suspicious MRI findings reducing also potential complications related to the procedure and all the consequence on quality of life (20-22). In our experience we have analyzed the impact of one of the targeted biopsy techniques, the MRI-US "fusion" prostate biopsy, in the diagnosis of clinically significant PCA; in particular the MRI-US "fusion" biopsy is simply described as a way to align a pre-registered MRI to an intra-procedure US in order to identify and target suspected lesions within the gland through a dedicated hardware platforms targeting areas found during mpMRI and not clearly visible during US scan. Based on our experience we can affirm that this technique had high sensitivity, accuracy and specificity than TRUS-GB and no significant difference for treatment zone between combined biopsies and targeted.

The advantages are the high reproducibility and the real time feedback though counterbalanced by the high upfront cost of the device; another advantage of the fusion technique is the ability to perform a systematic biopsy during the same session. In our series of patients the MRI-US fusion biopsy showed an elevate accuracy for diagnosis of csPCa; in particular the overall DR of csPCa was 69.1\% (65/94 cases); in particular we observed a statistically significant correlation between the PiRads V2 score and the presence of csPCa $(\mathrm{p}<0.001)$.

These results were in line with available studies in literature (23). Evaluating the detection rate of different techniques of targeted biopsy, Arsov et al. (24) compared the DR between an "in-bore" approach and a fusion approach: in particular they not observed important improvement in DR by fusion approach. In our previous experience we evaluated the role of "in-bore" MRI guided biopsy in a series of 70 patients (25); we observed an overall DR of $45.7 \%$ and in particular > 75\% in csPCa. Examining this series we observed an important correlation between the location of index lesion and the finding of PCA in the sample of biopsy. Venderlink et al. highlighted that there are no significant differences between magnetic resonance and fusion-guided biopsy; the only differences were related with an increasing lesion size (26). Considering the overall DR, in a NIH study of men with previous negative biopsy, a global DR of $37.4 \%$ using MRI fision biopsy was reported by Vourganti et al. (27). Other studies highlighted as performing 12 cores 
random biopsies the DR for csPCa is increasing respect to MRI fusion biopsy (28-30); in particular Radtke et al. reported that systematic transperineal prostate biopsy was more likely to miss Gleason > 7 PCA compared with MRI targeted biopsy (20.9\% vs $12.8 \%$ ) (30).

This dates are apparently in contrast with the results of our study; in fact the elevate DR for clinically significant disease depends on the fact that in addition to perform a standard 12-core biopsy we have added cores in areas normally not considered (lesions of the anterior and transitional); these data support the thesis about the essential role of mpMRI and MRI guided biopsy of suspicious lesions in the algorithm for evaluating men with previous negative TRUS-GB but with ongoing suspicion for PCA. Our study has some limitations: the number of patients, exclusive inclusion of patients with positive findings at mpMRI, no follow-up data and the lack of control group.

\section{Conclusions}

In conclusion, the results from our present study confirm that the mpMRI and MRI fusion guided biopsy have the purpose to improve detection of clinically significant PCA.

In particular the targeted MRI/TRUS fusion-guided biopsy represent a safe and accurate approach for diagnosis of csPCa, especially in patient with previous TRUS guided biopsy negative and suspicious PCA. Given that the experience of radiologist for mpMRI and of urologist for MRI/TRUS fusion-guided biopsy are critical, further studies are necessary to confirm these promising results.

\section{References}

1. American Cancer Society; Key Statistics for PCA. 2016.

2. Heidenreich A, Bastian PJ, Bellmunt J, et al. EAU guidelines on $P C A$, part 1: screening, diagnosis, and local treatment with curative intent-update 2013. Eur Urol 2014; 65:124-37.

3. Jones JS. Saturation biopsy for detecting and characterizing PCA. BJU Int 2007; 99:1340-4.

4. Lane BR, Zippe CD, Abouassaly R, et al. Saturation technique does not decrease cancer detection during follow up after initial prostate biopsy. J Urol. 2008; 179: 1746-50.

5. Dickinson L, Ahmed HU, Allen C, et al. Magnetic resonance imaging for the detection, localisation, and characterisation of prostate. Eur Urol. 2011; 59:477-94.

6. Testa C, Schiavina R, Lodi R, et al. Accuracy of MRI/MRSI-based transrectal ultrasound biopsy in peripheral and transition zones of the prostate gland in patients with prior negative biopsy. NMR Biomed 2010; 23:1017-26.

7. Baccos A, Schiavina R, Zukerman Z, et al. Accuracy of endorectal magnetic resonance imaging (MRI) and dynamic contrast enhanced-MRI (DCE-MRI) in the preoperative local staging of PCA. Urologia. 2012; 79:116-22.

8. Watanabe Y, Terai A, Araki T, et al. Detection and localization of PCA with the targeted biopsy strategy based on ADC map: a prospective large-scale cohort study. J Magn Reson Imaging 2012; 35:1414-21.

9. Rosenkrantz AB, Verma S, Choyke P, et al. Prostate magnetic resonance imaging and magnetic resonance imaging targeted biopsy in patients with a prior negative biopsy: a consensus statement by AUA and SAR. J Urol. 2016; 196:1613-8.

10. Wegelin O, van Melick HH, Hooft L, et al. Comparing three different techniques for magnetic resonance imaging-targeted prostate biopsies: a systematic review of in-bore versus magnetic resonance imaging-transrectal ultrasound fusion versus cognitive registration-is there a preferred technique. Eur Urol. 2017; 71:517-531

11. Filson CP, Natarajan S, Margolis DJ, et al. PCA detection with magnetic resonance-ultrasound fusion biopsy: the role of systematic and targeted biopsies. Cancer. 2016; 122:884-92.

12. Weinreb JC, Barentsz JO, Choyke PL, et al. PI-RADS prostate imaging - reporting and data system: 2015, version 2. Eur Urol. 2016; 69:16-40.

13. Schiavina R, Bianchi L, Borghesi M, et al. MRI displays the prostatic cancer anatomy and improves the bundles management before robot-assisted radical prostatectomy. J Endourol. 2018; 32:315321.

14. Vagnoni V, Bianchi L, Borghesi M, et al. Adverse features and competing risk mortality in patients with high-risk PCA. Clin Genitourin Cancer. 2017; 15:e239-e248.

15. Schiavina R, Chessa F, Borghesi M, et al. State-of-the-art imaging techniques in the management of preoperative staging and restaging of PCA. Int J Urol. 2019; 26:18-30.

16. Vagnoni V, Brunocilla E, Bianchi L, et al. State of the art of PET/CT with 11-choline and 18F-fluorocholine in the diagnosis and follow-up of localized and locally advanced PCA. Arch Esp Urol. 2015; 68:354-70

17. Schiavina R, Bianchi L, Mineo Bianchi F, et al. Preoperative staging with 11C-Choline PET/CT is adequately accurate in patients with very high-risk PCA. Clin Genitourin Cancer. 2018;16:305312.

18. Grasso AA, Cozzi G, DE Lorenzis E, et al. Multicenter analysis of pathological outcomes of patients eligible for active surveillance according to PRIAS criteria. Minerva Urol Nefrol. 2016; 68:237-41.

19. Schiavina R, Borghesi M, Brunocilla E, et al. The biopsy Gleason score $3+4$ in a single core does not necessarily reflect an unfavourable pathological disease after radical prostatectomy in comparison with biopsy Gleason score 3+3: looking for larger selection criteria for active surveillance candidates. PCA Prostatic Dis. 2015; 18:270-5.

20. Porreca A, Noale M, Artibani W, et al. Pros-IT CNR study group. Disease-specific and general health-related quality of life in newly diagnosed PCA patients: the Pros-IT CNR study. Health Qual Life Outcomes. 2018; 16:122.

21. Gacci M, Noale M, Artibani W, et al. Pros-IT CNR study group. Quality of Life After PCA Diagnosis: Data from the Pros-IT CNR. Eur Urol Focus. 2017; 3:321-324.

22. Noale M, Maggi S, Artibani W, et al. Pros-IT CNR study group. Pros-IT CNR: an Italian PCA monitoring project. Aging Clin Exp Res. 2017; 29:165-172.

23. Pinto PA, Ching PH, Rastinehad AR, et al. Magnetic resonance imaging /ultrasound fusion guided prostate biopsy improves cancer detection following transrectal ultrasound biopsy and correlates with multiparametric magnetic resonance imaging. J Urol. 2011; 186: 1281-5.

24. Arsov C, Rabenalt R, Blondin D, et al. Prospective randomized trial comparing magnetic resonance imaging (MRI)-guided in bore biopsy to MRI-ultrasound fusion and transrectal ultrasound guided 
prostate biopsy in patients with prior negative biopsies. Eur Urol. 2015; 68:713-20.

25. Schiavina R, Vagnoni V, D'Agostino D, et al. "In-bore" MRIguided prostate biopsy using an endorectal nonmagnetic device: a prospective study of 70 consecutive patients. Clin Genitourin Cancer. 2017; 15:417-427.

26. Venderlink W, Van der Leest M, Van Luijtelaar A, et al. Retrospective comparison of direct in-bore magnetic resonance imaging (MRI)-guided biopsy and fusion guided biopsy in patients with MRI lesions which are likely or highly likely to be clinically significant PCA. Word J Urol. 2017; 35:1849-1855.

27. Vourganti S, Rastinehad A, Yerram NK, et al. Multiparametric magnetic resonance imaging and ultrasound fusion biopsy detect PCA in patients with prior negative transrectal ultrasound biopsies. J Urol. 2012; 188: 2152-7.

28. Delongchamps NB, Peyromaure M, Schull A, et al. Prebiopsy magnetic resonance imaging and PCA detection: comparison of random and targeted biopsies. J Urol. 2013; 189: 493-930

29. Kuru TH, Saeb-Parsy K, Cantiani A, et al. Evolution of repeat prostate biopsy strategies incorporating transperineal and MRITRUS fusion techniques. World J Urol. 2014; 32: 945-5031.

30. Radtke JP, Kuru TH, Boxler S, et al. Comparative analysis of transperineal template-saturation prostate biopsy versus MRI-targeted biopsy with MRI-US fusion-guidance. J Urol. 2015; 193:87-94.

\section{Correspondence}

Daniele D'Agostino, MD (Corresponding Author)

dott.dagostino@gmail.com

Daniele Romagnoli, MD

Marco Giampaoli, MD

Paolo Corsi, MD

Alessandro Del Rosso, MD

Angelo Porreca, MD

Department of Robotic Urological Surgery, Abano Terme Hospital

Piazza Cristoforo Colombo 1, 35031 Abano Terme (PD) (Italy)

Federico Mineo Bianchi, MD

Riccardo Schiavina, MD

Eugenio Brunocilla, MD

Department of Urology, University of Bologna, Bologna (Italy) 